\title{
Derechos humanos de las mujeres en el mundo árabo-islámico: universalismo versus relativismo \\ (Women's human rights in the Arab-Islamic world: Universalism versus relativism)
}

OÑATI SOCIO-LEGAL SERIES VOLUME 10, ISSUE 1S (2020), 160S-183S: THE FOURTH WAVE OF FEMINISM: FROM SOCIAL NETWORKING AND SELF-DETERMINATION TO SISTERHOOD DOI LINK: HTTPS://DOI.ORG/10.35295/OSLS.IISL/0000-0000-0000-1086 RECEIVED 09 OCTOBER 2018, ACCEPTED 05 SEPTEMBER 2019

\section{EWA STRZELECKA* (D)}

\section{Resumen}

Este artículo hace una revisión crítica sobre la tensión y la posible reconciliación entre el relativismo y la universalidad de los derechos humanos de las mujeres en el mundo árabo-islámico. A través del análisis comparativo de los instrumentos internacionales, tales como la Convención sobre la Eliminación de Todas las Formas de Discriminación contra la Mujer (CEDAW), y los mecanismos regionales, tales como la Declaración de Derechos Humanos en el Islam y la Carta Árabe de Derechos Humanos, se destacan las brechas y los avances en la práctica de los derechos fundamentales de las mujeres en los veintidós Estados de la Liga Árabe. Como un ejemplo específico se debaten los cambios legislativos en materia de la transmisión de la nacionalidad por parte de las mujeres a sus hijos e hijas y a sus maridos extranjeros, conforme con la adaptación de la legislación nacional de los países árabo-islámicos a las exigencias de los tratados internacionales que garantizan la igualdad de género en el mundo.

\section{Palabras clave}

Derechos humanos de las mujeres; universalismo versus relativismo; CEDAW; Declaración de El Cairo sobre Derechos Humanos en el Islam; Carta Árabe de Derechos Humanos; Liga Árabe

\footnotetext{
Esta publicación se ha realizado en el marco del proyecto de investigación dirigido por la Dra. Laura Feliu Martínez y el Dr. Ferrán Izquierdo Brichs Dinámicas y actores transnacionales en Oriente Medio y Norte de África (MENA): Una genealogía histórica de élites y movimientos sociales entre lo local y lo global, Ministerio de Economía y Competitividad, Agencia Estatal de Investigación, y Fondo Europeo de Desarrollo Regional (HAR201677876-P), y en el marco del proyecto de investigación dirigido por el Dr. Ignacio Álvarez-Ossorio Alvariño Resiliencia del autoritarismo, choque de islamismos e intensificación del sectarismo en Oriente Medio y el Magreb, Ministerio de Economía y Competitividad - Secretaría de Estado de Investigación, Desarrollo e Innovación (CSO2017-86091-P).

* Ewa K. Strzelecka, Universidad de Granada. Avda. del Hospicio, s/n C.P. 18010 Granada. Dirección de email: ewaeva@ugr.es
} 


\section{Abstract}

This article critically examines the tension and possible reconciliation of competing views of culture relativism and the universality of women's human rights in the Arab-Islamic world. A comparative analysis of international human rights instruments, such as the Convention on the Elimination of All Forms of Discrimination against Women (CEDAW), and regional human rights protection mechanisms, such as the Cairo Declaration on Human Rights in Islam and the Arab Charter on Human Rights, allow us to get insight into the complexity of the cultural and legal definition and practice of women's rights within twenty-two States of the Arab League. Particular attention is paid to the national law amendments with regard to transmission of nationality from women to their children and foreign spouses. This is in accordance with the adaptation of the national legislation of the Arab-Islamic countries to the demands of international treaties which guarantee gender equality across the world.

\section{Key words}

Women's human rights; universalism versus relativism; CEDAW; Cairo Declaration on Human Rights in Islam; Arab Charter on Human Rights; Arab League 


\section{Table of contents}

1. Introduction. 1635

2. Derechos humanos de las mujeres: la CEDAW y la lógica argumentativa del universalismo versus relativismo cultural.

3. La CEDAW en el mundo árabo-islámico

4. Declaración de los Derechos Humanos en el Islam y la Carta Árabe de Derechos Humanos: mecanismos regionales de derechos humanos de las mujeres

5. Conclusiones.

Referencias. $179 S$

Referencias normativas $182 S$ 


\section{Introducción}

La cuestión de la incompatibilidad y la posible reconciliación entre el relativismo y la universalidad de los derechos humanos fundamentales ha producido una amplia literatura sobre esta temática (Donnelly 2013, Renteln 2013). El debate ha sido especialmente intenso en el contexto del mundo árabo-islámico (An-Na'im 2001, Sachedina 2009, Gutiérrez Castillo 2012, Mayer 2018), y en particular, en relación a los derechos humanos de las mujeres en el islam (Maquieira D'Angelo 1998, Ali 2000, Moghadam 2009, Cook 2012). El argumento comúnmente utilizado por los Estados árabo-islámicos para justificar las reservas a los tratados internacionales de los derechos humanos de las mujeres ha tenido que ver con su supuesta incompatibilidad con la sharía, la ley islámica. El término "sharía" significa literalmente "camino hacia un pozo de agua", que en la creencia musulmana se trata de la totalidad de la voluntad de Dios revelada al profeta Mahoma (Coulson 1964, Mir-Hosseini 2009, 26). La ruta del islam es una guía de conducta moral, que se percibe como algo sagrado, eterno y universal en las comunidades musulmanas (Mir-Hosseini 2009, 26). Hay dos ramas principales en el islam: el sunismo y el chiísmo, que aparecen ya en el siglo VII a raíz de las divisiones políticas en la primera comunidad musulmana, y que se rigen por diferentes escuelas de pensamiento jurídico (madhab). Las madhab principales en el islam suní son la hanafí, la malikí, la shafií y la hanbalí; y las madhab más importantes de la rama chiíta son la yafarí y la zaidí. Estas escuelas contribuyen a la jurisprudencia islámica (fiqh), a través de las interpretaciones específicas de la sharía llevada a cabo por un alfaquí, experto en fiqh (Coulson 1964, Engineer 2008, Strzelecka 2015). Cada Estado árabo-islámico establece en su Constitución el grado de aplicación de la sharía en su legislación nacional. Normalmente, la diferencia consiste en que la sharía es la fuente única o es una de las fuentes de legislación interna. En el primer caso, la sharía es la fuente principal y la más importante de todo el ordenamiento jurídico. En el segundo caso, la sharía se aplica con más rigurosidad respecto a determinadas regulaciones del derecho penal y sobre todo en las Leyes de la Familia y los Códigos de Estatuto Personal.

Este artículo hace una revisión crítica de la tensión entre el universalismo de los derechos humanos y el relativismo cultural, que apuesta por una definición particular de los derechos de acuerdo con la cultura y la religión de cada pueblo. El análisis de los instrumentos internacionales, tales como la Convención sobre la Eliminación de Todas las Formas de Discriminación contra la Mujer (CEDAW), y de los mecanismos regionales, específicamente de la Declaración de Derechos Humanos en el Islam y la Carta Árabe de Derechos Humanos, permite destacar las brechas y los avances en la práctica de los derechos humanos de las mujeres en el mundo árabo-islámico. Esta investigación se centra en la consideración de estos instrumentos en los 22 Estados de la Liga Árabe: Argelia, Egipto, Sudán, Arabia Saudí, Marruecos, Libia, Yemen, Siria, Yibuti, Irak, Somalia, Túnez, Emiratos Árabes Unidos, Comoras, Jordania, Líbano, Palestina, Omán, Kuwait, Mauritania, Qatar y Bahréin. Actualmente todos estos Estados, con la excepción de Arabia Saudí, han codificado la sharía en las Leyes de la Familia o de Estatuto Personal, de acuerdo con la exégesis de la escuela de jurisprudencia predominante en su territorio. Estos códigos reconocen la diferencia en derechos y responsabilidades entre hombres y mujeres, y perpetúan leyes que son discriminatorias en términos de género. Las interpretaciones de la sharía en la clave patriarcal y su uso al servicio del poder político han sido ampliamente criticadas por varias corrientes del 
feminismo, incluido el feminismo islámico que emerge desde finales de los años ochenta (Badran 2009). Autoras tales como Amina Wadud, Asma Barlas, Azizah al-Hibri, Riffat Hassan, Ziba Mir-Hosseini y Qudsiyya Mirza, entre otras, se han dedicado a deconstruir la visión patriarcal del islam por medio de una metodología basada en el pensamiento crítico. Sus perspectivas femeninas y feministas en la interpretación de las fuentes sagradas del islam han tenido como objetivo la promoción de la justicia y la igualdad de género en los contextos musulmanes. Este trabajo ha contribuido de forma significativa a la reflexión sobre la universalidad de los derechos humanos en un marco compatible con los valores islámicos.

\section{Derechos humanos de las mujeres: la CEDAW y la lógica argumentativa del universalismo versus relativismo cultural}

Los derechos humanos se consideran una realidad dinámica. Este hecho ha sido especialmente relevante para la evolución de los derechos humanos de las mujeres en el mundo. Gracias al movimiento revitalizador feminista y su denuncia de la discriminación de género, se lograron ampliar el concepto y el goce de los derechos humanos a través del reconocimiento de los derechos humanos específicos de las mujeres. La Convención sobre la Eliminación de Todas las Formas de Discriminación contra la Mujer (CEDAW), aprobada por la Asamblea General de las Naciones Unidas en diciembre de 1979, y que entró en vigor el 3 de septiembre de 1981, se considera la carta magna de los derechos humanos de las mujeres del mundo. Su importancia radica en el carácter jurídicamente vinculante y en el hecho de ser la recopilación más completa de derechos civiles, políticos, sociales, económicos, culturales, además de los derechos colectivos y del desarrollo de las mujeres. Al recoger en un único instrumento todos estos derechos, la CEDAW fortalece el concepto de indivisibilidad de los derechos humanos. No obstante, la CEDAW ha sido también objeto de críticas en relación a su posicionamiento universalista por partidarios de posturas cercanas al relativismo cultural. En respuesta a este relativismo, las mujeres de diferentes regiones del mundo debatieron la idea de reafirmar los derechos de las mujeres como parte inherente de los derechos humanos universales en 1985, en el Foro de las ONG de la III Conferencia Internacional sobre las Mujeres en Nairobi (Hodgson 2003, 6). Las alianzas, las redes y la movilización feminista a nivel global hicieron posible comprometer a las Naciones Unidas para que se reconociera formalmente este hecho en la II Conferencia Mundial de los Derechos Humanos de Viena en 1993. El párrafo 18 de la Declaración y el Programa de Acción de Viena (1993) estipula: "Los derechos humanos de la mujer y de la niña son parte inalienable, integrante e indivisible de los derechos humanos universales".

A pesar de los avances en los foros internacionales, los defensores del relativismo no reconocen la universalidad de los derechos humanos. Argumentan que los tratados internacionales de los derechos humanos han sido definidos por las agencias internacionales que representan unos valores específicos, es decir, los occidentales. Su postura es que la representación del mundo, las creencias, los valores y las prácticas de cada grupo humano deben ser definidos, interpretados y aplicados de acuerdo con los parámetros de su contexto cultural. En esta línea se ha justificado la necesidad de la emergencia de los mecanismos regionales de los derechos humanos. En el contexto del mundo árabo-islámico se han establecido dos mecanismos regionales que representan específicamente una visión islámica y árabe de los derechos humanos: la Declaración de 
los Derechos Humanos en el Islam, promulgada de 1990, y la Carta Árabe de Derechos Humanos, revisada en 2004 (Rishmawi 2005). Además, los Estados árabo-islámicos, miembros de la Unión Africana, hacen referencia a la Carta Africana de Derechos Humanos y de los Pueblos, conocida como Carta de Banjul, promulgada en 1987 (Viljoen 2009). Estos países, con excepción de Egipto, Marruecos y Túnez, han firmado o ratificado también el Protocolo a la Carta Africana de Derechos Humanos y de los Pueblos, más conocido como Protocolo de Maputo, promulgado en 2003, y en vigor desde 2005.

TABLA 1

\begin{tabular}{|c|c|c|c|c|c|c|}
\hline \multirow[t]{2}{*}{ Estado } & \multicolumn{3}{|c|}{$\begin{array}{l}\text { Carta Africana de Derechos } \\
\text { Humanos y de los Pueblos (1981) }\end{array}$} & \multicolumn{3}{|c|}{ Protocolo de Maputo (2003) } \\
\hline & Firmado & Ratificado & Depositado & Firmado & Ratificado & Depositado \\
\hline Argelia & $10 / 04 / 1986$ & 01/03/1987 & 20/03/1987 & $29 / 12 / 2003$ & & \\
\hline Comoras & $07 / 12 / 2004$ & 01/06/1986 & 18/07/1986 & 26/02/2004 & $18 / 03 / 2004$ & $16 / 04 / 2004$ \\
\hline Yibuti & 20/12/1991 & $11 / 11 / 1991$ & 20/12/1991 & $18 / 12 / 2003$ & $02 / 02 / 2005$ & $04 / 02 / 2005$ \\
\hline Egipto & $16 / 11 / 1981$ & 20/03/1984 & 03/04/1984 & \multicolumn{3}{|c|}{ No firmado ni ratificado. } \\
\hline Libia & $30 / 05 / 1985$ & 19/07/1986 & 26/03/1987 & $05 / 11 / 2003$ & $23 / 05 / 2004$ & $30 / 06 / 2004$ \\
\hline Mauritania & $25 / 02 / 1982$ & $14 / 06 / 1986$ & 26/06/1986 & & $21 / 09 / 2005$ & $14 / 12 / 2005$ \\
\hline Marruecos* & \multicolumn{3}{|c|}{ No firmado ni ratificado. } & \multicolumn{3}{|c|}{ No firmado ni ratificado. } \\
\hline RASD $^{* 1}$ & $10 / 04 / 1986$ & 02/05/1986 & 23/05/1986 & $20 / 06 / 2006$ & & \\
\hline Túnez & & $16 / 03 / 1983$ & $22 / 04 / 1983$ & \multicolumn{3}{|c|}{ No firmado ni ratificado. } \\
\hline Sudán & 03/09/1982 & $18 / 02 / 1986$ & $11 / 03 / 1986$ & $30 / 06 / 2008$ & & \\
\hline $\begin{array}{l}\text { República de } \\
\text { Sudán del Sur* }\end{array}$ & \multicolumn{3}{|c|}{ No firmado ni ratificado. } & $24 / 01 / 2013$ & & \\
\hline Somalia & 26/02/1982 & $31 / 07 / 1985$ & 20/03/1986 & $23 / 02 / 2006$ & & \\
\hline
\end{tabular}

Tabla 1: Estados árabo-islámicos de la Unión Africana Partes de la Carta Africana de Derechos Humanos y de los Pueblos (1981) y el Protocolo de Maputo (2003).

(Fuente: Elaboración propia, con datos de ACHPR 2019a, 2019b.)

Los defensores del universalismo argumentan que los derechos humanos hacen referencia a los valores de la dignidad humana y al respeto para los seres humanos que están presentes en todas las tradiciones éticas y religiosas del mundo. La Declaración de los Derechos Humanos en el Islam y la Carta Árabe de Derechos Humanos, basadas en los valores musulmanes y árabes, reconocen la mayoría de estos principios. Su

\footnotetext{
${ }^{1}$ La República Árabe Saharaui Democrática (RASD) es el Estado parcialmente reconocido, que desde 1984 forma parte de la Unión Africana (UA). No obstante, ni la Liga Árabe ni la ONU reconocen a la RASD como un Estado soberano. Debido al conflicto entre Marruecos y el Frente Polisario, Marruecos se salió de la Unión Africana en protesta por la admisión de la RASD. Treinta y dos años después, Marruecos fue readmitido el 30-01-2017 como Estado-miembro de la UA.
} 
inconsistencia con los derechos humanos universales tiene que ver con los aspectos que cuestionan las estructuras de poder en el sistema dominante. La reivindicación de la diferencia cultural que resiste las reformas a favor de la igualdad de género protege los intereses del régimen patriarcal. En este sentido hay que preguntarse hasta qué punto la cultura, o la utilización política de la cultura, se convierten en un poderoso instrumento de legitimación de la discriminación contra las mujeres, porque "es precisamente en nombre de la cultura, es decir, el discurso sobre la suprema importancia de preservar y salvar la especificidad cultural, el que hoy se presenta como el más potente a nivel planetario como forma de desactivar los reclamos y demandas de las mujeres en el mundo que vivimos" (Maquieira D'Angelo 1998, 187). La antropóloga Virginia Maquieira D'Angelo (1998) habla del "culturalismo sexista" o el "sexismo cultural", que opera como una nueva forma de fundamentalismo cultural. Se trata de una postura que justifica la resistencia a los cambios de género y a los avances en los derechos humanos universales de las mujeres en base a las reivindicaciones relacionadas con la especificidad cultural.

La idea de los derechos de las mujeres como derechos humanos universales desafía al relativismo cultural e invoca un ámbito de justicia y de ética global. Los defensores del universalismo argumentan que los principios de la igualdad y la dignidad humana, sobre los que se construye la idea de los derechos humanos, están por encima de la especificidad de la discriminación cultural y religiosa. La CEDAW (1979) se constituye como un instrumento jurídico para promover la igualdad sustantiva y obligar a los Estados Partes a respetar, proteger y garantizar los derechos humanos de las mujeres de todo el mundo. Se enumeran, además, varias razones por las que el enfoque de derechos contribuye a promover el empoderamiento de las mujeres. En primer lugar, los derechos humanos universales ponen límites a la supremacía de todos los que, por su posición social, política o de género, ejercen y abusan del "poder sobre" (Rowlands 1997). Los derechos humanos contribuyen a la expansión de las libertades y las capacidades de las personas. No solamente reconocen la dignidad de las mujeres, sino que también reafirman que sus derechos específicos son indivisibles, inalienables y universales, lo que significa que nadie puede limitarlos, ni acabar con ellos, y que representan valores transculturales. Varias autoras (Hodgson 2003, Strzelecka 2009) hablan de la utilidad de este enfoque para negociar con el Estado las reformas legislativas a favor de la igualdad y promover una conciencia social sobre los derechos humanos de las mujeres. La CEDAW tiene especial relevancia en este sentido, como un instrumento jurídicamente vinculante, para evocar la responsabilidad del Estado signatario a que cumpla con sus obligaciones. Obliga a los Estados Partes a respetar, proteger y garantizar los derechos de las mujeres y erradicar la discriminación de género, así como adoptar medidas temporales de acción afirmativa. ${ }^{2}$ Amplía también la responsabilidad estatal a determinados actos cometidos por personas privadas, empresas o instituciones no estatales.

\footnotetext{
2 Según la CEDAW, la discriminación contra las mujeres se define como: "toda distinción, exclusión o restricción basada en el sexo que tenga por objeto o por resultado menoscabar o anular el reconocimiento, goce o ejercicio por la mujer, independientemente de su estado civil, sobre la base de la igualdad del hombre y la mujer, de los derechos humanos y las libertades fundamentales en las esferas política, económica, social, cultural y civil o en cualquier otra esfera" (art. 1).
} 
Los Estados que han ratificado la CEDAW deben promover una serie de políticas para asegurar el pleno desarrollo y adelanto de las mujeres, con el objetivo de garantizar el ejercicio y el goce de sus derechos humanos y las libertades fundamentales, en igualdad de condiciones con los hombres (art. 3). Con este fin, el artículo 4 aconseja la adopción de acciones afirmativas, como medidas especiales de carácter temporal, encaminadas a acelerar la igualdad de oportunidades y de trato entre las mujeres y los hombres. La CEDAW presta atención también a las funciones reproductivas de las mujeres, y destaca el apoyo a las medidas especiales encaminadas a proteger la maternidad (art. 4.2). Entre otras disposiciones, compromete a los Estados Partes a proteger los derechos humanos de las mujeres a través de las medidas que erradiquen la trata de blancas y la explotación de la prostitución (art. 6); promuevan la eliminación de la discriminación de las mujeres en la vida política y pública (art. 7); promocionen su participación y representación en la esfera internacional (art. 8); garanticen la no discriminación en cuanto a la nacionalidad (art. 9); promuevan la igualdad de derechos en el ámbito de la educación (art. 10); en el empleo (art. 11); en la atención médica (art. 12); en las prestaciones económicas y sociales (art. 13), en las actividades de esparcimiento, deportes y en todos los aspectos de la vida cultural (art.13.c); además de promocionar a las mujeres rurales (art. 14); y garantizar la igualdad ante la ley (art. 15), y en los asuntos relacionados con el matrimonio y las relaciones familiares (art. 16). Para conseguir la plena realización de estos derechos de la CEDAW, se incita a los Estados a promover unas políticas adecuadas en el ámbito nacional (art. 24), que incluyan medidas de carácter legislativo, administrativo y de otras índoles, con el fin de modificar o derogar las leyes, reglamentos, usos y prácticas que son discriminatorias para las mujeres (art. $2 \mathrm{f}$ ).

La CEDAW permite la ratificación sujeta a reservas, siempre que las reservas no sean incompatibles con el objeto y el propósito de la Convención. En la práctica, hay reservas tan generales que pueden afectar significativamente a las disposiciones fundamentales de la CEDAW. Entre los factores que se han opuesto al pleno reconocimiento de los derechos universales de las mujeres están los argumentos de índole cultural y religiosa, así como la superposición de la soberanía estatal. Hay también obstáculos a la institucionalidad y las limitaciones en doctrina legal de los derechos humanos. Algunos gobiernos, además, han tratado de justificar su pasividad respecto a la violencia doméstica contra las mujeres por una supuesta limitación del poder público para intervenir en los asuntos que pertenecen al ámbito privado de las personas. No obstante, en los últimos años el éxito del argumento feminista de que lo personal es político ha permitido avanzar en esta materia. Como consecuencia, muchos de los Estados Partes de la CEDAW han promulgado leyes en contra de la violencia de género y han tomado medidas especiales para proteger a las víctimas y penalizar a los agresores.

La parte más polémica de la CEDAW tiene que ver con su posicionamiento universalista frente a la crítica del relativismo cultural. Reconoce que la cultura y las tradiciones pueden jugar un rol en el mantenimiento de la discriminación contra las mujeres y obliga a los Estados a eliminar los estereotipos en los roles de hombres y mujeres. La línea argumentaria se basa en la postura de que no se pueden invocar en nombre de la cultura aquellas tradiciones y prácticas culturales que discriminen, estereotipen, humillen, subordinen y marginen a las mujeres, así como las que se oponen al logro de la plena igualdad con los hombres. La CEDAW va más allá y no solamente propone la revisión crítica de las costumbres y las tradiciones, sino que también compromete a los Estados 
Partes a tomar medidas estratégicas con el fin de modificar y erradicar aquellos elementos culturales que puedan limitar el ejercicio de los derechos fundamentales de las mujeres. Su visión, inscrita en el preámbulo, implica que "para lograr la plena igualdad entre los hombres y las mujeres es necesario modificar el papel tradicional tanto de los hombres como de las mujeres en la sociedad y en la familia". Esto se traduce en el requerimiento de "modificar los patrones socioculturales de conducta de hombres y mujeres, con miras a alcanzar la eliminación de los prejuicios y las prácticas consuetudinarias, y de cualquier otra índole, que estén basadas en la idea de la inferioridad o superioridad de cualquiera de los sexos o en funciones estereotipadas de hombres y mujeres" (art. 5). Con este fin, se recomienda el rediseño de libros, programas escolares y métodos de enseñanza, desde una perspectiva de género, y la promoción de estímulos para una educación mixta, con el fin de eliminar los conceptos estereotipados sobre los roles masculinos y femeninos en el sistema educativo (art. 10 c). Estas recomendaciones son especialmente polémicas y criticadas por los fundamentalismos religiosos y los grupos ultraconservadores que en nombre de los derechos culturales y el respeto a sus creencias reclaman la preservación de los modelos tradicionales de género y la separación por sexo en los espacios educativos u otros. Aunque no podemos considerar estos grupos reaccionarios como los únicos que emplean argumentos enraizados en el relativismo cultural.

Otro desafío de la CEDAW está en que la Convención no faculta al Comité para recibir denuncias ni iniciar investigaciones, sino para hacer recomendaciones a los Estados en base al análisis de los informes periódicos. Para superar estas limitaciones la Asamblea General de la ONU adoptó el 6 de octubre de 1999 el Protocolo Facultativo de la CEDAW, que entró en vigor el 22 de diciembre de 2000. Es un documento que introduce el mecanismo de la denuncia de la violación por los Estados Partes de los derechos estipulados en la CEDAW (art. 1). Las comunicaciones pueden ser presentadas por "personas o grupos de personas que se hallen bajo la jurisdicción del Estado Parte y que aleguen ser víctimas de una violación por ese Estado Parte de cualquiera de los derechos enunciados en la Convención, o en nombre de esas personas o grupos de personas" (art. 2). El Protocolo faculta también al Comité CEDAW para iniciar su propia investigación si recibe información fidedigna sobre violaciones graves o sistemáticas por un Estado Parte respecto a los derechos de las mujeres estipulados en la CEDAW (art. 8). Cuando se justifique y con el consentimiento del Estado Parte, la investigación podrá incluir una visita a su territorio (art. 8.2). En cualquier caso "el Comité invitará a ese Estado Parte a colaborar en el examen de la información y, a esos efectos, a presentar observaciones sobre dicha información" (art. 8.1). El Artículo 10 considera, no obstante, que todo Estado Parte podrá, al momento de la firma o ratificación del Protocolo, o de la adhesión a él, declarar que no reconoce la competencia del Comité establecida en el artículo 8, lo cual debilita la fuerza de la convención.

\section{La CEDAW en el mundo árabo-islámico}

Veinte de los veintidós países de la Liga Árabe son Estados Partes de la CEDAW. Sudán y Somalia son los únicos Estados del mundo árabo-islámico que no lo son. No obstante, tan solo Comoras, Yibuti y el Estado de Palestina han aceptado la CEDAW sin ninguna condición. Los demás 17 países de los 20 Estados Partes mantienen reservas a la Convención. Arabia Saudí y Emiratos Árabes Unidos han firmado y han ratificado la 
CEDAW, pero no han puesto en marcha los instrumentos de adhesión. De los veinte Estados Partes de la CEDAW, tan solo dos firmaron el Protocolo Facultativo de la CEDAW: Libia en 2004 y Túnez en 2008.

TABLA 2

\begin{tabular}{|c|c|c|c|c|}
\hline & Estado & $\begin{array}{l}\text { CEDAW: } \\
\text { ratificación, } \\
\text { adhesión (a) o } \\
\text { sucesión (d) }\end{array}$ & $\begin{array}{l}\text { CEDAW: } \\
\text { reservas }\end{array}$ & $\begin{array}{l}\text { Protocolo Facultativo } \\
\text { CEDAW: ratificación, } \\
\text { adhesión (a) o sucesión }(\mathrm{d})\end{array}$ \\
\hline 1. & Argelia & 22 mayo 1996 (a) & Sí & No \\
\hline 2. & Bahréin & 18 junio 2002 (a) & Sí & No \\
\hline 3. & Comoras & 31 octubre 1994(a) & No & No \\
\hline 4. & Yibuti & 2 diciembre 1998 (a) & No & No \\
\hline 5. & Egipto & 18 septiembre 1981 & Sí & No \\
\hline 6. & Iraq & 13 agosto 1986 (a) & Sí & No \\
\hline 7. & Jordania & 1 julio 1992 & Sí & No \\
\hline 8. & Kuwait & 2 septiembre 1994 (a) & Sí & No \\
\hline 9. & Líbano & 21 abril 1997 (a) & Sí & No \\
\hline 10. & Libia & 16 mayo 1989 (a) & Sí & Sí, 18 junio 2004 (a) \\
\hline 11. & Mauritania & 10 mayo 2001 (a) & Sí & No \\
\hline 12. & Marruecos & 21 junio 1993 (a) & Sí & No \\
\hline 13. & Omán & 7 febrero 2006 (a) & Sí & No \\
\hline 14. & Palestina & 2 abril $2014(\mathrm{a})$ & No & No \\
\hline 15. & Qatar & 29 abril 2009 (a) & Sí & No \\
\hline 16. & Arabia Saudí & 7 septiembre 2000 & Sí & No \\
\hline 17. & Siria & 28 marzo 2003 (a) & Sí & No \\
\hline 18. & Túnez & 20 septiembre 1985 & Sí & Sí, 23 septiembre 2008 (a) \\
\hline 19. & EAU & 6 octubre 2004 & Sí & No \\
\hline 20. & Yemen & 30 mayo $1984(a)$ & Sí & No \\
\hline 21. & Sudán & No & - & No \\
\hline 22. & Somalia & No & - & No \\
\hline
\end{tabular}

Tabla 2. Estados árabo-islámicos partes de la CEDAW y del Protocolo Facultativo de la CEDAW.

(Fuente: elaboración propia con datos de CEDAW, ONU 2018.)

Los Estados suelen justificar las reservas a la CEDAW sobre la base de la soberanía estatal y la regla de la superioridad de las leyes nacionales, la tradición, la religión o la cultura. En el mundo árabo-islámico, 12 Estados Partes utilizaron el pretexto de la sharía para destacar el supuesto conflicto entre algunas disposiciones de la Convención y la ley islámica, considerada superior por su origen divino. Las reservas a los artículos 
específicos de la CEDAW por sus contradicciones con la sharía las formularon: Bahréin, Egipto, Iraq, Kuwait, Libia, Mauritania, Marruecos, Siria, Emiratos Árabes y Qatar. Omán y Arabia Saudí, además de las reservas específicas, hicieron una reserva general por la ley islámica, dificultando de forma significativa la implementación de la CEDAW en la práctica. La mayoría de las reservas a la CEDAW realizadas por los Estados áraboislámicos conciernen a los siguientes artículos: artículo 2 de la Convención sobre las medidas de la política pública a favor de la igualdad y en contra de la discriminación; artículo 9 sobre la nacionalidad; artículo 15 sobre la igualdad ante la ley; y artículo 29 sobre el procedimiento de solución de controversias entre Estados Partes.

Los artículos 2 y 16 son considerados por el Comité de CEDAW como disposiciones fundamentales para los fines y el propósito de la Convención. El artículo 2 compromete a los Estados Partes a condenar la discriminación contra las mujeres en todas sus formas y adoptar las estrategias, las acciones y las medidas legislativas, constitucionales y de otro carácter para erradicar esta discriminación. Obliga a los Estados Partes a abstenerse en todo acto de prácticas de discriminación, a establecer la protección jurídica y efectiva de los derechos de las mujeres, a modificar las leyes, los reglamentos, los usos y las prácticas que constituyan discriminación de género. La reserva a este artículo cuestiona, por lo tanto, de forma significativa el fin de la Convención, especialmente si partimos del principio de que los Estados que ratifican la CEDAW lo hacen porque consideran que la discriminación contra las mujeres debe ser eliminada. Es la razón por la que el Comité de CEDAW está particularmente preocupado por el número y el alcance de las reservas formuladas a este artículo. Hay nueve Estados árabo-islámicos (Siria, Bahréin, Qatar, Emiratos Árabes, Argelia, Egipto, Iraq, Marruecos y Libia) que han hecho la reserva específica al artículo 2. Omán y Arabia Saudí también pueden objetar este artículo por la reserva general en referencia a su incompatibilidad con la sharía.

El artículo 9 de la CEDAW obliga a los Estados Partes a otorgar a las mujeres iguales derechos que a los hombres para adquirir, cambiar o conservar su nacionalidad, y transferirla a sus hijos. El artículo 9 garantiza que ni el matrimonio con un extranjero ni el cambio de nacionalidad del marido durante el matrimonio cambien automáticamente la nacionalidad de la esposa, la convierta en apátrida o la obligue a adoptar la nacionalidad del cónyuge. Hay nueve Estados de la Liga Árabe que mantienen la reserva a este artículo: Siria, Bahréin, Qatar, Emiratos Árabes, Omán, Arabia Saudí, Kuwait, Líbano y Jordania. En realidad, las leyes de nacionalidad que son discriminatorias para las mujeres existen en veinte Estados de la Liga Árabe, de los cuales 18 son parte de la CEDAW. Tan solo en Túnez y en Yibuti las mujeres gozan del mismo derecho que los hombres para conferir la nacionalidad tanto a sus hijos o hijas como a su esposo extranjero.

A fecha de hoy (2019), tan solo en siete Estados árabo-islámicos (Túnez, Marruecos, Egipto, Argelia, Comoras, Yibuti y el Estado de Palestina) las mujeres casadas con extranjeros pueden conferir la ciudadanía a sus hijos o hijas. En todos ellos, con la excepción de Túnez y Yibuti, la desigualdad de género persiste en cuanto a la adquisición de la nacionalidad por matrimonio. En marzo de 2018, Marruecos anunció la preparación de una reforma para permitir a las mujeres conferir la nacionalidad marroquí a su esposo extranjero tal y como se permite a los hombres marroquíes en relación con sus esposas forasteras (The North African Post 2018). No obstante, más de 
un año después (octubre 2019) no se ha avanzado en la implementación de esta propuesta para garantizar la plena igualdad de género en la transmisión de la nacionalidad marroquí. Hasta ahora las enmiendas de mejora para erradicar o disminuir la discriminación de las mujeres en cuanto a la transmisión de su nacionalidad se han dado en diez países: Egipto en 2004, Argelia en 2005, Marruecos en 2007, Túnez en 1993 y en 2010, Palestina en 2003, y reformas parciales en Iraq en 2006, en Sudan en 2006, en Yemen en 2008, en Libia en 2010 y en Omán en 2014. En Líbano, en 2018 se anunció una propuesta de reforma de la ley de ciudadanía que permitiría a las mujeres pasar la nacionalidad a sus hijos o hijas. No obstante, la propuesta ha sido criticada por las organizaciones de mujeres, porque no contempla el disfrute de este derecho para las mujeres que están casadas con los nacionales de los países vecinos, es decir, los sirios y los palestinos (Sewell 2018). En Siria, se ha presentado una enmienda de la ley de nacionalidad en 2010 para garantizar un derecho igualitario a las mujeres en la cuestión de la nacionalidad y la descendencia, pero el estallido de la guerra paralizó los trabajos sobre la reforma.

Lo que preocupa es que el pleno derecho de las mujeres a transferir la nacionalidad a sus hijos o hijas no está reconocido en 16 Estados árabo-islámicos: Bahréin, Iraq, Jordania, Kuwait, Líbano, Mauritania, Omán, Qatar, Arabia Saudí, Somalia, Sudán, Siria, Emiratos Árabes Unidos, Yemen y Libia. Los países con las leyes de ciudadanía más discriminatorias, que permiten solo a los hombres conferir la nacionalidad a sus hijos o hijas, son Qatar y Somalia. La ley de Kuwait es igualmente restrictiva, aunque en teoría se contempla una excepción muy limitada. Se permite a las madres cuyo hijo o hija es de padre desconocido transferir su nacionalidad, siempre y cuando el Ministerio del Interior, a su discreción, tome esta medida extraordinaria, lo cual raramente ocurre en la práctica (UNHCR 2017). En Jordania, Libia, Arabia Saudí, Bahréin y Emiratos Árabes no se permite a las mujeres casadas con los extranjeros conferir la nacionalidad a sus hijos o hijas, pero existen circunstancias específicas en las que se reconoce este derecho a las mujeres. Esto ocurre cuando el padre del hijo o hija es desconocido, apátrida, de nacionalidad desconocida o porque no reconozca su paternidad (UNHCR 2017). En Yemen, tras la reforma de la ley de nacionalidad de 2008, se aplica una ley similar, es decir se reconoce el derecho de las madres a conferir la nacionalidad a sus hijos o hijas si el padre del hijo o hija es desconocido o apátrida (UNICEF 2011, 2, Strzelecka 2017). En Siria, las madres pueden conferir la nacionalidad solamente si el niño o niña nació en Siria y el padre no reconoce su paternidad. Siria tiene también una salvaguarda para prevenir el riesgo de apatridia entre los niños nacidos en su territorio, pero no está claro si esto se implementa en la práctica (UNHCR 2017, 4). En Líbano, de forma similar, se establecen procedimientos excepcionales que permiten a las madres conferir la nacionalidad, pero solo si el hijo o hija nace fuera del matrimonio (UNHCR 2017).

Hay países árabo-islámicos que reconocen el derecho de las mujeres a conferir la nacionalidad a sus hijos o hijas, aunque este derecho no está garantizado en condiciones de plena igualdad con los hombres. Así, en Iraq las mujeres pueden conferir la nacionalidad a su prole, pero el ejercicio de este derecho se dificulta cuando se trata de los hijos o hijas de madres iraquíes y de padres desconocidos o apátridas, y que nacieron en el extranjero. Para tales nacimientos, el artículo 4 de la Ley de nacionalidad iraquí, permite al hijo o hija solicitar la nacionalidad de su madre dentro de un año desde el alcance de su mayoría de edad y siempre cuando resida en Iraq en el momento de 
solicitud. La ley de nacionalidad omaní de 2014 reconoce el derecho de transmisión de la nacionalidad por la madre, pero pide garantías adicionales, es decir, que el padre de la mujer sea omaní o apátrida. Tan solo en el caso de que el padre del hijo o hija sea desconocido, se exime a la madre de la comprobación de la identidad de origen. En Mauritania, las madres pueden conferir la nacionalidad a sus hijos o hijas cuando el padre es desconocido, apátrida o extranjero, pero el hijo o hija tiene derecho a renunciar a esta nacionalidad un año antes de alcanzar la mayoría de edad, incluso si esto le convierte en apátrida. Los niños nacidos en el extranjero de madres mauritanas y padres extranjeros pueden optar por la nacionalidad mauritana un año antes de alcanzar la mayoría de edad.

El artículo 16 de la CEDAW establece que los Estados Partes deben adoptar todas las medidas adecuadas para eliminar la discriminación contra las mujeres en todos los asuntos relacionados con el matrimonio y las relaciones familiares y, en particular, asegurar, en condiciones de igualdad entre hombres y mujeres, "el mismo derecho para contraer matrimonio; el mismo derecho para elegir libremente cónyuge y contraer matrimonio sólo por su libre albedrío y su pleno consentimiento; los mismos derechos y responsabilidades durante el matrimonio y con ocasión de su disolución; los mismos derechos y responsabilidades como progenitores, cualquiera que sea su estado civil, en materias relacionadas con sus hijos; en todos los casos, los intereses de los hijos serán la consideración primordial; los mismos derechos a decidir libre y responsablemente el número de sus hijos y el intervalo entre los nacimientos y a tener acceso a la información, la educación y los medios que les permitan ejercer estos derechos; los mismos derechos y responsabilidades respecto de la tutela, curatela, custodia y adopción de los hijos, o instituciones análogas cuando quiera que estos conceptos existan en la legislación nacional; en todos los casos, los intereses de los hijos serán la consideración primordial; los mismos derechos personales como marido y mujer, entre ellos el derecho a elegir apellido, profesión y ocupación; los mismos derechos a cada uno de los cónyuges en materia de propiedad, compras, gestión, administración, goce y disposición de los bienes, tanto a título gratuito como oneroso" (art. 16). Además, el artículo 16 considera que "no tendrán ningún efecto jurídico los esponsales y el matrimonio de niños y se adoptarán todas las medidas necesarias, incluso de carácter legislativo, para fijar una edad mínima para la celebración del matrimonio y hacer obligatoria la inscripción del matrimonio en un registro oficial".

En 14 países árabo-islámicos (Siria, Bahréin, Qatar, Emiratos Árabes, Omán, Argelia, Egipto, Iraq, Marruecos, Kuwait, Líbano, Libia, Jordania y Mauritania) se mantienen las reservas específicas al artículo 16 de la CEDAW. Arabia Saudí, al mantener una reserva general en base a la sharía, también limita de forma significativa la implementación de este artículo. El conflicto entre el artículo 16 de la CEDAW y la sharía se puede dar por la interpretación conservadora de la ley islámica en las cuestiones de la poliginia, el divorcio unilateral masculino (talaq), la obligación de manutención que tiene el marido respecto a su esposa (nafaqa), la obligación de la sumisión de la mujer a su esposo, el matrimonio temporal (mutah), la pubertad islámica versus la edad mínima del matrimonio anunciada en la CEDAW, la desigualdad de género en cuanto a la herencia y al valor del testimonio, y la perpetuación del modelo tradicional de familia sustentado sobre la división de los roles de género. 
El artículo 15 de la CEDAW compromete a los Estados Partes a garantizar la igualdad de las mujeres con los hombres ante la ley. Hay 7 Estados árabo-islámicos (Siria, Bahréin, Qatar, Emiratos Árabes, Omán, Argelia y Marruecos) que mantienen reservas específicas a este artículo. Arabia Saudí también puede objetarlo por su reserva general sobre la base de la sharía. El artículo 15 reconoce a las mujeres, en materias civiles, una capacidad jurídica idéntica a la de los hombres y las mismas oportunidades para el ejercicio de esa capacidad. Los Estados Partes deben, por lo tanto, reconocer a las mujeres iguales derechos para firmar contratos y administrar bienes y dispensarle un trato igual en todas las etapas del procedimiento en las cortes de justicia y en los tribunales. Además, deben reconocer a los hombres y a las mujeres los mismos derechos con respecto a la legislación relativa al derecho de las personas a circular libremente y a la libertad para elegir su residencia y domicilio. El último punto suscita controversias particulares en las comunidades conservadoras del mundo árabo-islámico por el tema de la libertad de movimientos de las mujeres y la eliminación de su control por los hombres.

Hay que reconocer también que en los últimos años se han dado algunas mejoras significativas en el mundo árabo-islámico en cuanto a los avances de los derechos de las mujeres, que se han manifestado en la retirada de las reservas u objeciones a la CEDAW por algunos Estados. El éxito más relevante es que todos los Estados de la Liga Árabe que mantenían la reserva al artículo 7 sobre la vida política y pública, la han retirado, reconociendo de esta forma el pleno uso de los derechos políticos de las mujeres. Positivamente, Túnez, en 2014 retiró la mayoría de sus reservas a la CEDAW, dejando únicamente la reserva general que anuncia que la CEDAW no se aplicará si contradice el capítulo 1 de la Constitución tunecina que considera que: “Túnez es un Estado libre, independiente y soberano, su religión es el islam, su lengua el árabe y su régimen la república" (art. 1). Mauritania ha retirado también parcialmente las reservas a la CEDAW en 2014, manteniendo la reserva general sobre la base de su posible incompatibilidad con la sharía. Este avance se puede clasificar como un cambio cosmético, porque la interpretación tradicional de la ley islámica supone un desafío en cuanto a la práctica de las disposiciones de los derechos humanos universales.

TABLA 3

\begin{tabular}{|c|c|c|c|c|c|c|}
\hline País & $\begin{array}{l}\text { Art. } 2 \\
\text { Medidas } \\
\text { de } \\
\text { política } \\
\text { pública }\end{array}$ & $\begin{array}{l}\text { Art. } 9 \\
\text { Nacionalidad }\end{array}$ & $\begin{array}{l}\text { Art. } 15 \\
\text { Igualdad } \\
\text { ante la ley }\end{array}$ & $\begin{array}{l}\text { Art. } 16 \\
\text { Matri- } \\
\text { monio y } \\
\text { familia }\end{array}$ & \begin{tabular}{|l|} 
Art. 29 \\
Mecanismo de \\
solución de \\
controversias \\
entre Estados \\
partes
\end{tabular} & $\begin{array}{l}\text { Otras } \\
\text { reservas }\end{array}$ \\
\hline $\begin{array}{l}\text { Siria } \\
\text { Bahréin } \\
\text { Qatar } \\
\text { Emiratos } \\
\text { Árabes }\end{array}$ & $x$ & $x$ & $x$ & $x$ & $x$ & \\
\hline Omán & & $x$ & $x$ & $x$ & $x$ & $\begin{array}{l}\text { x (reserva } \\
\text { general por } \\
\text { la sharía). }\end{array}$ \\
\hline Arabia Saudí & & $x$ & & & $x$ & $\begin{array}{l}\text { x (reserva } \\
\text { general por } \\
\text { la sharía). }\end{array}$ \\
\hline
\end{tabular}




\begin{tabular}{|c|c|c|c|c|c|c|}
\hline País & $\begin{array}{l}\text { Art. } 2 \\
\text { Medidas } \\
\text { de } \\
\text { política } \\
\text { pública }\end{array}$ & \begin{tabular}{|l} 
Art. 9 \\
Nacionalidad
\end{tabular} & $\begin{array}{l}\text { Art. } 15 \\
\text { Igualdad } \\
\text { ante la ley }\end{array}$ & $\begin{array}{l}\text { Art. 16 } \\
\text { Matri- } \\
\text { monio y } \\
\text { familia }\end{array}$ & \begin{tabular}{|l} 
Art. 29 \\
Mecanismo de \\
solución de \\
controversias \\
entre Estados \\
partes
\end{tabular} & $\begin{array}{l}\text { Otras } \\
\text { reservas }\end{array}$ \\
\hline Argelia & $x$ & & $x$ & $x$ & $x$ & \\
\hline \begin{tabular}{|l|} 
Egipto \\
Iraq \\
\end{tabular} & $x$ & & & $x$ & $x$ & \\
\hline Marruecos & $x$ & & $x$ & & $x$ & \\
\hline $\begin{array}{l}\text { Kuwait } \\
\text { Líbano }\end{array}$ & & $x$ & & $x$ & $x$ & \\
\hline Libia & $x$ & & & $x$ & & \\
\hline Jordania & & $x$ & & $x$ & & \\
\hline Mauritania & & & & $x$ & & Art. 13 \\
\hline Yemen & & & & & $x$ & \\
\hline Túnez & & & & & & $\begin{array}{l}\text { x (reserva } \\
\text { general por } \\
\text { el capítulo } 1 \\
\text { de la } \\
\text { Constitución) } \\
\end{array}$ \\
\hline
\end{tabular}

Tabla 3. Estados de la Liga Árabe: reservas a la CEDAW.

(Fuente: elaboración propia, a partir de datos de ONU 2018.)

\section{Declaración de los Derechos Humanos en el Islam y la Carta Árabe de Derechos Humanos: mecanismos regionales de derechos humanos de las mujeres}

La emergencia de los mecanismos regionales de los derechos humanos se ha justificado argumentando que los derechos humanos deben ser definidos de acuerdo con la cultura, la religión y la necesidad de cada pueblo. En esta línea, la Organización para la Cooperación Islámica, la organización intergubernamental más grande que representa a los Estados islámicos, promulgó en 1990 la Declaración de los Derechos Humanos en el Islam (DDHI), conocida también como Declaración de El Cairo. La declaración proporciona una visión general de la perspectiva musulmana sobre los derechos humanos y fija la sharía como su fuente principal. Su definición de los derechos humanos relativiza los estándares internacionales respecto a la igualdad de género en varios aspectos. El artículo 6 de la Declaración estipula que las mujeres son iguales a los hombres en dignidad humana, sin embargo, sus derechos y deberes son diferentes. Aunque no se específica en qué consisten estas diferencias, se declara que "el marido es responsable de la manutención y bienestar de la familia" (art. 6 b). Desde el lado positivo, la DDHI reconoce el derecho de las mujeres a su propia entidad civil y autonomía financiera (art. 6), el derecho a conservar su nombre y linaje (art. 6), el derecho al matrimonio (art. 5), y a ser protegida en el caso de conflictos armados (art. 4). No se mencionan otros derechos, o se hace de una forma que resulta bastante controvertida. Por ejemplo, el artículo 12 estipula que "el hombre tiene derecho, en el marco de la sharía, a la libre circulación y a la elección de su lugar de residencia, ya sea dentro o fuera de su país". La forma en la que se estipula esta disposición, según las voces críticas, abre la posibilidad de restringir legalmente el movimiento de las mujeres, como se 
acostumbra a hacer en algunos países musulmanes, en base a las tradiciones patriarcales y a las interpretaciones ultraconservadores del islam. Las divergencias y la relativización de la plena igualdad de género hacen que la DDHI se contradiga, en muchos aspectos, con los derechos humanos universales de las mujeres, y que desafíe los estandartes internacionales en esta materia.

\section{TABLA 4}

\begin{tabular}{|c|c|c|c|c|}
\hline & \multirow[t]{2}{*}{ Estado } & \multirow{2}{*}{$\begin{array}{l}\text { Declaración } \\
\text { de los } \\
\text { Derechos } \\
\text { Humanos en } \\
\text { el Islam (1990) }\end{array}$} & \multicolumn{2}{|c|}{ Carta Árabe de Derechos Humanos (2004) } \\
\hline & & & Firmado & Ratificado \\
\hline 1. & Argelia & Sí & 02.08 .2004 & 11.06.2006 \\
\hline 2. & Bahréin & Sí & 05.07.2005 & 18.06.2006 \\
\hline 3. & Comoras & Sí & - & - \\
\hline 4. & Yibuti & Sí & - & - \\
\hline 5. & Egipto & $\mathrm{Si}$ & 05.09 .2004 & - \\
\hline 6. & Iraq & Sí & 19.11.2010 & 04.04 .2013 \\
\hline 7. & Jordania & Sí & 28.10.2004 & 28.10.2004 \\
\hline 8. & Kuwait & Sí & 18.09.2006 & - \\
\hline 9. & Líbano & Sí & 25.09.2006 & 08.05.2011 \\
\hline 10. & Libia & Sí & 14.02 .2005 & 07.08 .2006 \\
\hline 11. & Mauritania & Sí & - & - \\
\hline 12. & Marruecos & Sí & 27.12.2004 & \\
\hline 13. & Omán & Sí & - & - \\
\hline 14. & Palestina & Sí & 15.07.2004 & 28.11.2007 \\
\hline 15. & Qatar & Sí & 24.01.2008 & 11.01.2009 \\
\hline 16. & Arabia Saudí & Sí & 01.08 .2004 & 15.04.2009 \\
\hline 17. & Siria & Sí & 17.08.2006 & 06.02 .2007 \\
\hline 18. & Túnez & Sí & 15.06.2004 & - \\
\hline 19. & EAU & Sí & 18.09.2006 & 15.01.2008 \\
\hline 20. & Yemen & Sí & 12.10.2004 & 12.11.2008 \\
\hline 21. & Sudan & Sí & 21.07.2005 & - \\
\hline 22. & Somalia & Sí & - & - \\
\hline
\end{tabular}

Algo más completa en cuanto a los derechos de las mujeres es la Carta Árabe de Derechos Humanos, propuesta por la Liga Árabe en 1994, revisada y modificada en 2004, y que entró en vigor el 15 de marzo 2008. Se trata de un instrumento regional de los 
derechos humanos, que se considera especialmente relevante debido a su posible impacto en el mundo árabo-islámico. La Carta Árabe de Derechos Humanos de 2004 está compuesta por 53 artículos. El preámbulo hace referencia a los valores religiosos y culturales de la nación árabe, y particularmente a su "fe en la dignidad de la persona" y al "derecho humano a una vida decente basada en la igualdad, justicia e igualdad". El islam es la única religión nombrada específicamente en el documento, mientras que otras creencias se mencionan de manera general como "religiones divinamente reveladas", sin especificar a las minorías religiosas, cuya presencia es relevante en los países de la Liga Árabe. Más de 20 millones de cristianos, de diferentes ramas, viven en países tales como Líbano, Egipto, Irak, Bahréin, Siria, Kuwait y Jordania. Hay también comunidades judías, drusas, bahá'ís, gnósticas e hindúes, entre otros. La diversidad cultural de los países de la Liga Árabe se constituye por varios grupos étnicos y en una variedad de idiomas. Por ejemplo, en países tales como Argelia, Libia, Marruecos y Túnez, destaca la presencia considerable del pueblo amazigh (bereber) que convive con el árabe. En Yibuti, los árabes, los etíopes y los europeos constituyen tan solo 5\% de la población, mientras que los grupos mayoritarios son los somalís (60\%) y los afar (35\%). Aparte de varios dialectos de la lengua árabe, entre los idiomas oficiales del mundo árabe-islámico están el tamazigh (bereber), el somalí y el kurdo.

El gran avance de la Carta Árabe está en su intención de superar el relativismo cultural y adaptar los principios árabes a la universalidad de los derechos humanos. Un paso en esta dirección se ha dado mediante el reconocimiento de ciertos estándares internacionales, tales como la admisión de que "todos los derechos humanos son universales, indivisibles, interdependientes y están relacionados entre sí" (art. 1.4). Otro progreso legal es el hecho de que la Carta Árabe no hace referencias directas al relativismo cultural, religioso o de otra índole, y deriva sus valores directamente de los tratados internacionales. Por un lado, hace referencia a los principios de la Carta de las Naciones Unidas, la Declaración Universal de los Derechos Humanos (DUDH), el Pacto Internacional de Derechos Civiles y Políticos (PDCP), y el Pacto Internacional de Derechos Económicos, Sociales y Culturales (PDESC). Por otro lado, tiene en cuenta también la Declaración de El Cairo sobre Derechos Humanos en el Islam (DDHI). Por lo general, la Carta Árabe de Derechos Humanos se considera consistente con los estándares internacionales de los derechos humanos en muchos aspectos, no obstante, cuando revisamos en detalle sus disposiciones respecto a los derechos de las mujeres, nos encontramos con limitaciones y contradicciones importantes que se exponen más adelante.

El valor positivo de la Carta Árabe es su referencia al principio de la igualdad. Su objetivo general es reforzar los valores de "la igualdad, la tolerancia y la moderación" de las nuevas generaciones (art. 1.3), lo cual suscribe una apuesta en contra de la radicalización de la sociedad y del fundamentalismo religioso. Es más, el artículo 3.3 reconoce que "los hombres y las mujeres son iguales en respeto de la dignidad humana", y que los Estados Partes deben adoptar todas las medidas necesarias para "garantizar la igualdad de oportunidades y la igualdad efectiva entre los hombres y las mujeres en el goce de todos los derechos enunciados en la Carta". El documento hace referencia también a las acciones afirmativas a favor de las mujeres. No obstante, su comprensión de estas medidas difiere considerablemente de las conceptualizaciones internacionales, y en particular de la definición de la CEDAW. El artículo 4 de la CEDAW sobre las 
acciones positivas indica: "La adopción por los Estados partes de medidas especiales de carácter temporal encaminadas a acelerar la igualdad de facto entre el hombre y la mujer no se considerará discriminación en la forma definida en la presente Convención, pero de ningún modo entrañará, como consecuencia, el mantenimiento de normas desiguales o separadas; estas medidas cesarán cuando se hayan alcanzado los objetivos de igualdad de oportunidad y trato".

La Carta Árabe habla de una igualdad basada en derechos y obligaciones diferentes entre los hombres y las mujeres, definidas según "el marco de una discriminación positiva establecida a favor de las mujeres por la sharía, ley islámica, y otras leyes divinas, así como por las leyes y los instrumentos jurídicos aplicables" (art. 3.3). Además, añade que las "leyes vigentes son las que regulan los derechos y los deberes del hombre y de la mujer en cuanto al matrimonio, durante el matrimonio y en el caso de su disolución" (art. 33.1). La referencia al marco de las leyes religiosas y la legislación nacional en asuntos de la familia y el estatuto personal hacen que la Carta Árabe falle en reconocer que precisamente estas son las leyes que las feministas identifican como un obstáculo para el reconocimiento de la ciudadanía plena de las mujeres y la igualdad de género. Estas leyes se basan en una interpretación patriarcal de la sharía y ponen el énfasis en las diferencias de los derechos y las obligaciones entre mujeres y hombres, y favorecen el poder masculino sobre ellas. Es difícil, por lo tanto, considerar estas medidas como una "discriminación positiva", o una acción afirmativa en terminología feminista, cuando en realidad se trata de una discriminación negativa, que no socava, sino que refuerza la discriminación y la desigualdad de género.

La Carta Árabe de los Derechos Humanos representa un ejemplo de la preocupación por tres elementos relevantes en la cultura árabe: la familia, el matrimonio y la maternidad. Según el artículo 33.1: "la familia es el elemento natural y fundamental de la sociedad, y se basa en el matrimonio entre un hombre y una mujer". Entre los avances positivos, se encuentra la disposición sobre el requerimiento del consentimiento libre y pleno de la novia y del novio para poder casarse (art. 33.1). Esta disposición contribuye a la lucha contra los matrimonios forzados, no obstante, no aborda el problema de los matrimonios precoces. La Carta Árabe no fija una edad mínima para el matrimonio, y tan solo estipula que "los hombres y las mujeres en edad de casarse tienen derecho a contraer el matrimonio y fundar una familia" (art. 33.1). Este artículo tiene que ver con la interpretación tradicional de la sharía, en la que se considera la pubertad como el momento que marca la transición de la infancia hacia la edad adulta que capacita a la persona a contraer el matrimonio. Tradicionalmente, las autoridades religiosas explicaban que el casamiento de las hijas en una edad temprana ayuda a evitar la tentación de la zina, es decir, una relación fuera del matrimonio que en el islam se considera ilícita y que está penalizada. La CEDAW, al contrario, prohíbe el matrimonio de menores y estipula que los Estados Partes deben fijar la edad mínima, normalmente a los 18 años, para que la celebración del matrimonio tenga efectos jurídicos y sea inscrita en el registro oficial.

La protección de las madres, como uno de los grupos vulnerables, es otra de las preocupaciones centrales de la Carta Árabe de los Derechos Humanos (art. 33.2). La protección de la maternidad se aborda no tanto en la clave del bienestar centrado en las mujeres, sino por el interés mayor de los y las niñas. En este sentido se establece, por 
ejemplo, que "la pena de muerte no se impondrá a la mujer embarazada antes del parto o en el período de lactancia de dos años después del parto; en todos los casos, el mejor interés del niño o niña debe ser considerado primordialmente" (art. 7.2). Esta disposición es un ejemplo que se contradice con los derechos humanos internacionales, ya que no condena la pena de muerte, sino que la pospone. Por eso, fue criticada por la comunidad internacional como una norma que anula el derecho primordial a la vida, por contemplar la posibilidad de la pena de muerte. Las discrepancias de la Carta Árabe con los derechos humanos universales se dan también en los casos relativos a la nacionalidad. El artículo 9.2 de la CEDAW garantiza la igualdad en la transmisión de la nacionalidad a los niños y niñas, sin discriminación en base al sexo. La Carta Árabe, en cambio, relativiza este hecho y permite a los Estados Partes adoptar las "medidas que consideran apropiadas, de conformidad con su legislación interna en materia de nacionalidad, para permitir que un niño o una niña adquiera la nacionalidad de la madre, teniendo en cuenta, en todo caso, el mejor interés del niño o la niña" (art. 29.2). Las voces críticas señalan que esta disposición falla, al reconocer que las leyes nacionales de la mayoría de los Estados árabo-islámicos no dotan a las mujeres del mismo derecho que a los hombres para transferir su nacionalidad a los hijos o hijas. La discriminación legal contra las mujeres se mantiene también en relación a las cuestiones del matrimonio, el divorcio, el testimonio, el acceso a la propiedad, la custodia de los hijos o hijas, la sucesión y la herencia.

Entre los elementos positivos de la Carta Árabe de Derechos Humanos está la prohibición explícita de la violencia contra las mujeres. El artículo 33.2 de la Carta estipula: "El Estado y la sociedad deben garantizar la protección de la familia, el fortalecimiento de los vínculos familiares, la protección de sus miembros, y la prohibición de todas las formas de violencia o de abuso en las relaciones entre sus miembros, y en particular contra las mujeres y los niños" (art. 33.2). La prohibición de la violencia y del abuso contra las mujeres es un avance de gran relevancia, a pesar de que la Carta restringe su área de actuación exclusivamente al ámbito familiar. Otro avance positivo de la Carta Árabe de Derechos Humanos es la proclamación de la igualdad entre mujeres y hombres en cuanto al derecho al empleo, a la formación, a la protección laboral, y a una remuneración igual por un trabajo igual (art. 34.4). Se reconoce y visibiliza también el rol de las mujeres en el desarrollo, urgiendo a los Estados Partes a "adoptar las medidas pertinentes para garantizar la colaboración entre hombres y mujeres con el fin de lograr los objetivos nacionales del desarrollo" (art. 41.3). Se considera, además, que las disposiciones de la Carta Árabe no deben menoscabar los derechos y las libertades de las mujeres que habían sido garantizados en la legislación nacional (art. 43).

En virtud del artículo 45, se establece un Comité de los Derechos Humanos Árabes, compuesto por siete personas expertas y elegidas cada cuatro años. El primer Comité se formó en marzo de 2009 (Rishmawi 2010, 172). Su objetivo ha sido el de revisar y hacer recomendaciones en respuesta a los informes que los Estados Partes preparan cada tres años, en relación a sus progresos en la implementación de esta Carta (art. 48). El Comité está comprometido a publicar sus informes de forma anual, con lo cual sus comentarios y recomendaciones para los gobiernos están disponibles para el conocimiento ciudadano. No obstante, no se contemplan las investigaciones y los mecanismos de queja en el caso de las violaciones de los derechos humanos que se establecen en la Carta 
Árabe, lo cual constituye una debilidad para proceder y reivindicar la rendición de cuentas. A pesar de sus limitaciones, la Carta Árabe representa un esfuerzo importante para aproximarse a los estándares internacionales respecto a la transparencia y los derechos humanos universales. Es también un ejemplo que nos introduce en el debate sobre la especificidad cultural versus los derechos humanos universales.

\section{Conclusiones}

La práctica de los derechos humanos está garantizada por los tratados internacionales y el contrato social entre el Estado y la ciudadanía. La práctica deseada de estos derechos depende de que el enfoque de derechos no sea vaciado de sus contenidos, ni instrumentalizado al uso de los intereses políticos y económicos, o relativizado por los enfoques culturalistas. El reconocimiento de los derechos de las mujeres como derechos humanos universales representa no solamente una larga historia de lucha por la justicia de género, sino también un avance en la articulación de la ética global, basada en los valores humanistas de dimensiones universales. Los enfoques culturalistas, no obstante, no reconocen esta universalidad de los derechos humanos y ponen el énfasis en la particularidad y la diferencia cultural. El análisis de las reservas a la CEDAW por los veintidós Estados árabo-islámicos, la Declaración de El Cairo sobre Derechos Humanos en el Islam y la Carta Árabe de Derechos Humanos, ponen en evidencia cómo los derechos humanos de las mujeres han quedado debilitados, entre otras cuestiones, en nombre de la cultura y la ley islámica. En este contexto, hay que preguntarse quién, por qué y para qué se interpreta la sharía y decidir sobre su compatibilidad con los derechos humanos universales. Las tradiciones y las costumbres locales, las estructuras sociales y económicas, así como las políticas estatales y la agencia de las personas, son factores relevantes para comprender los modos en los que se conforman los hechos religiosos a nivel individual y colectivo en cada contexto y en cada momento histórico. Para entender las realidades sociales de los países árabo-islámicos no debemos ver el islam como el único factor explicativo y de más relevancia con respecto a otros factores sociales, económicos y políticos, que influyen en la vida de las mujeres. Es importante volver la atención de la figura teórica del patriarcado hacia los procesos dinámicos en los que las mujeres y los hombres participan, reproduciendo, o por el contrario, desafiando y subvirtiendo los regímenes de género dominantes, y sus mecanismos de legitimación, en los contextos históricos, políticos, sociales y económicos específicos. En este sentido la integración de las mujeres en el discurso de los derechos humanos en un plano de igualdad con los hombres ha supuesto un avance esencial y al mismo tiempo ha visibilizado los desafíos pendientes y las resistencias al cambio social y político necesario para la igualdad de género. Estos retos no son exclusivos de los Estados de la Liga Árabe, sino que existen en todo el mundo.

\section{Referencias}

ACHPR, 2019a. Ratification Table: African Charter on Human and Peoples' Rights [en línea]. Banjul: African Commission on Human and Peoples' Rights. Disponible en: https://www.achpr.org/ratificationtable?id=49 [Con acceso el 18 de junio de 2019].

ACHPR, 2019b. Ratification Table: Protocol to the African Charter on Human and Peoples' Rights on the Rights of Women in Africa [en línea]. Banjul: African Commission on 
Human and Peoples' Rights. Disponible en:

https://www.achpr.org/ratificationtable?id=37 [Con acceso el 18 de junio de 2019].

Ali, S.S., 2000. Gender and Human Rights in Islam and International Law. Leiden: Brill.

An-Na'im, A., 2001. Human Rights in the Arab World: A Regional Perspective. Human

Rights Quarterly [en línea], 23(3), 701-732. Disponible en:

https://doi.org/10.1353/hrq.2001.0026 [Con acceso el 18 de junio de 2019].

Badran, M., 2009. Feminism in Islam: Secular and Religious Convergences. Oxford:

Oneworld.

Cook, R.J., ed., 2012. Human Rights of Women: National and International Perspectives. Filadelfia: University of Pennsylvania Press.

Coulson, N.J., 1964. A History of Islamic Law. Nueva York: Routledge.

Donnelly, J., 2013. Universal Human Rights in Theory and Practice. Ithaca: Cornell University Press.

Engineer, A.A., 2008. Rights of Women in Islam. Nueva Delhi: Sterling.

Gutiérrez Castillo, V.L., 2012. Estados Árabes y Derechos Humanos: la Recepción y Aplicación de la Norma Internacional. Revista Española de Derecho Internacional [en línea], LXIV(2), 105-131. Disponible en: http://redi.ene-estudio.es/wpcontent/uploads/2017/09/04-GUTIERREZ_digital.pdf [Con acceso el 18 de junio de 2019].

Hodgson, D., 2003. Women's Rights as Human Rights: Women in Law and Development in Africa. Africa Today [en línea], 49(2), 1-26. Disponible en: https://doi.org/10.1353/at.2003.0010 [Con acceso el 14 de octubre de 2019].

International Center for Not-for-Profit Law, 2019. Civic Freedom Monitor: League of Arab States [en línea]. Washington, DC: International Center for Not-for-Profit Law. Disponible en: http://www.icnl.org/research/monitor/las.html [Con acceso el 18 de junio de 2019].

Maquieira D'Angelo, V., 1998. Cultura y Derechos Humanos de las Mujeres. En: P. Pérez Cantó, ed., Las mujeres del Caribe en el umbral del 2000. Comunidad de Madrid, Dirección General de la Mujer.

Mayer, A.E., 2018. Islam and Human Rights [en línea]. Nueva York: Routledge.

Disponible en: https://doi.org/10.4324/9780429495120 [Con acceso el 14 de octubre de 2019].

Mir-Hosseini, Z., 2009. Towards Gender Equality: Muslim Family Laws and the Shari' ah. En: Z. Anwar, ed., Wanted: Equality and Justice in the Muslim Family. Selangor: Musawah, 23-63.

Moghadam, V.M., 2009. Feminism, Legal Reform and Women's Empowerment in the Middle East and North Africa. International Social Science Journal [en línea], 59, 916. Disponible en: https://doi.org/10.1111/j.1468-2451.2009.00673.x [Con acceso el 18 de junio de 2019].

Naciones Unidas, 2018. Los redactores de la Declaración Universal de los Derechos Humanos [en línea]. ONU. Disponible en: https://www.un.org/es/sections/universal- 
declaration/drafters-universal-declaration-human-rights/index.html [Con acceso el 18 de junio de 2019].

Renteln, A.D., 2013. International Human Rights: Universalism versus Relativism. Nueva Orleans: Quid Pro Books.

Rishmawi, M., 2005. The Revised Arab Charter on Human Rights: A Step Forward? Human Rights Law Review [en línea], 5(2), 361-376. Disponible en: http://doi.org/10.1093/hrlrev/ngi021 [Con acceso el 18 de junio de 2019].

Rishmawi, M., 2010. The Arab Charter on Human Rights and the League of Arab States: An Update. Human Rights Law Review [en línea], 10(1), 169-178. Disponible en: https://doi.org/10.1093/hrlr/ngp043 [Con acceso el 18 de junio de 2019].

Rowlands, J., 1997. Questioning Empowerment: Working with Women in Honduras [en línea]. Oxford: Oxfam. Disponible en: https://doi.org/10.3362/9780855988364 [Con acceso el 18 de junio de 2019].

Sachedina, A., 2009. Islam and the Challenge of Human Rights [en línea]. Oxford University Press. Disponible en: https://doi.org/10.1093/acprof:oso/9780195388428.001.0001 [Con acceso el 18 de junio de 2019].

Sewell, A., 2018. Lebanon nationality law set to change, but critics say doesn't go far enough. Middle East Eye [en línea], 3 de abril. Disponible en: https://www.middleeasteye.net/news/lebanon-nationality-law-1732890336 [Con acceso el 14 de octubre de 2019].

Strzelecka, E., 2009. Género, desarrollo y diversidad cultural. En: E. Molina Bayón y N. San Miguel, eds., Nuevas líneas de investigación en género y desarrollo. Universidad Autónoma de Madrid, 99-148.

Strzelecka, E., 2015. Las mujeres en la tradición islámica y el uso del poder interpretativo del Corán. En: J. Ruiz Arévalo, ed., Manual de asesor de género en operaciones. Granada: Tleo.

Strzelecka, E., 2017. Mujeres en la Primavera Árabe: Construcción de una cultura política de resistencia feminista en Yemen. Madrid: Consejo Superior de Investigaciones Científicas (CSIC).

The North Africa Post, 2018. Moroccan Women will soon Pass Nationality to Foreign Spouses. The North Africa Post [en línea], 18 de marzo. Disponible en: http://northafricapost.com/22747-moroccan-women-will-soon-pass-nationalityforeign-spouses.html [Con acceso el 14 de octubre de 2019].

UNHCR, 2017. Background Note on Gender Equality: Nationality Laws and Statelessness [en línea]. Disponible en: http://www.refworld.org/pdfid/58aff4d94.pdf [Con acceso el 18 de junio de 2019].

UNICEF, 2011. Yemen: MENA Gender Equality Profile: Status of Girls and Women in the Middle East and North Africa. Informe. Nueva York: UNICEF.

Viljoen, F., 2009. An Introduction to the Protocol to the African Charter on Human and Peoples' Rights on the Rights of Women in Africa. Washington and Lee Journal of 
Civil Rights and Social Justice [en línea], 16(1), 11-46,

https://scholarlycommons.law.wlu.edu/crsj/vol16/iss1/4 [Con acceso el 14 de octubre de 2019].

\section{Referencias normativas}

Arab Charter on Human Rights [en línea]. Liga de los Estados Árabes, 22 de mayo de 2004. Disponible en: http://hrlibrary.umn.edu/instree/loas2005.html [Con acceso el 18 de junio de 2019].

Carta africana sobre los derechos humanos y de los pueblos (carta de Banjul) [en línea].

Nairobi: XVIII Asamblea de Jefes de Estado y Gobierno de la Organización de Unidad Africana, 27 de julio de 1981. Disponible en:

http://www.acnur.org/fileadmin/Documentos/BDL/2002/1297.pdf?view=1 [Con acceso el 18 de junio de 2019].

Carta de las Naciones Unidas [en línea]. San Francisco, 26 de junio de 1945. Disponible en: http://www.un.org/es/charter-united-nations/ [Con acceso el 18 de junio de 2019].

Convención sobre la eliminación de todas las formas de discriminación contra la mujer

(Resolución 34/180) (CEDAW) [en línea]. Nueva York: Naciones Unidas, 18 de diciembre de 1979. Disponible en:

https://www.ohchr.org/sp/professionalinterest/pages/cedaw.aspx [Con acceso el 18 de junio de 2019].

Declaración de los Derechos Humanos en el Islam [en línea]. Conferencia Islámica de El

Cairo, 1990. Disponible en: http://www.refworld.org/cgi-

bin/texis/vtx/rwmain/opendocpdf.pdf?reldoc=y\&docid=50acbf1c2 [Con acceso el 18 de junio de 2019].

Declaración sobre el derecho al desarrollo (Res. 41/128) [en línea]. Nueva York: Asamblea

General de Naciones Unidas, 4 de diciembre de 1986. Disponible en:

https://www.ohchr.org/SP/ProfessionalInterest/Pages/RightToDevelopment.aspx

[Con acceso el 18 de junio de 2019].

Declaración Universal de los Derechos Humanos [en línea]. París, 10 de diciembre de1948.

Disponible en: http://www.un.org/es/universal-declaration-human-rights/ [Con acceso el 18 de junio de 2019].

Declaración y Programa de Acción de Viena [en línea]. Conferencia Mundial de Derechos Humanos, 25 de junio de 1993. Disponible en:

https://www.ohchr.org/Documents/Events/OHCHR20/VDPA_booklet_Spanish.p df [Con acceso el 18 de junio de 2019].

Pacto Internacional de Derechos Civiles y Políticos (res. 2200 A (XXI)) [en línea]. Nueva York: Asamblea General de Naciones Unidas, 16 de diciembre de 1966.

Disponible en: https://www.ohchr.org/sp/professionalinterest/pages/ccpr.aspx [Con acceso el 18 de junio de 2019].

Pacto Internacional de Derechos Económicos, Sociales y Culturales (res. 2200 A (XXI) [en línea]. Nueva York: Asamblea General de Naciones Unidas, 16 de diciembre de 1966. Disponible en: 
https://www.ohchr.org/sp/professionalinterest/pages/cescr.aspx [Con acceso el 18 de junio de 2019].

Protocol to the African Charter on Human and Peoples' Rights on the Rights of Women in Africa (Maputo Protocol) [en línea]. Maputo, 11 de julio de 2003. Disponible en: https://www.un.org/en/africa/osaa/pdf/au/protocol_rights_women_africa_2003.p df [Con acceso el 18 de junio de 2019].

Protocolo Facultativo de la Convención sobre la eliminación de todas las formas de discriminación contra la mujer (A/RES/54/4) [en línea]. Nueva York: Naciones Unidas, 6 de octubre de 1999. Disponible en:

https://www.ohchr.org/sp/professionalinterest/pages/opcedaw.aspx [Con acceso el 18 de junio de 2019]. 